Case Report

\title{
Stercoral perforation: A rare case and
} review

\author{
Lava Krishna Kannappa*, Muhammad Sufian Khalid, May Hnin \\ Lwin Ko, Mohsin Hussein, Jia Hui Choong, Ameer Omar Rawal- \\ Pangarkar, Danaradja Armugam and Yahya Salama
}

Kettering General Hospital, United Kingdom

\section{Abstract}

We present a 54-year-old male with abdominal pain, Vomiting and weight loss since 5 months. Perforation was noted at recto-sigmoid junction and underwent Hartman's procedure with end colostomy. Histology of sigmoid colon confirmed a Stercoral perforation without any evidence of dysplasia or malignancy. Patient had chemotherapy for squamous cell carcinoma (SCC) of epiglottis a year ago and was on codeine phosphate and Oromorph as and when required since his treatment for SCC for pain. Patient also had been suffering from constipation since he finished chemotherapy. Stercoral perforation always need to kept in mind in patients who present with constipation and need to take all patients who present with chronic constipation and initiate measures we encounter commonly in everyday practice. We present a brief review about Stercoral perforation and its management.

\section{More Information}

*Address for Correspondence: Lava Krishna Kannappa, MD, Kettering General Hospital, United Kingdom, Tel: 00447912759193; Email: lavakrishna@yahoo.com

Submitted: 14 October 2019 Approved: 26 October 2019 Published: 28 October 2019

How to cite this article: Kannappa LK, Khalid MS, Lwin Ko MH, Hussein M, Choong JH, et al. Stercoral perforation: A rare case and review. Arch Cancer Sci Ther. 2019; 3: 049-051.

DOI: dx.doi.org/10.29328/journal.acst.1001009 ORCiD: orcid.org/0000-0003-2792-8653

Copyright: @ 2019 Kannappa LK, et al. This is an open access article distributed under the Creative Commons Attribution License, which permits unrestricted use, distribution, and reproduction in any medium, provided the original work is properly cited

Check for updates

\section{Case Summary}

54-year-old male presented with one-day history of worsening abdominal pain, vomiting dysuria and weight loss of about 4 stones since 5 months. Past history included squamous cell carcinoma of epiglottis and had been treated with radiotherapy and chemotherapy a year prior to admission and had PEG tube removed 3 months prior to this admission. Patient was on codeine phosphate and oromorph for pain on and off since his chemotherapy ended a year ago. He used to open up his bowels normally prior to chemotherapy, But Post Chemotherapy he used to open his bowels once or twice a week and was on laxatives started by his general practitioner. On examination, His abdomen was tender in lower abdomen, especially in right iliac fossa region with guarding. His observation showed a blood pressure of $110 / 70 \mathrm{~mm}$ of $\mathrm{Hg}$, Heart rate of 81 per minute, Respiratory rate of 20 per minute, Saturations of $96 \%$ on air and a temperature of 36.90 centigrade.

Blood results were normal apart from raised white cell count of 13.1, Neutrophils of 12.3, C-reactive protein of 168 and Blood lactate of 2.5. Initial chest and abdominal radiography did not show any free sub-diaphragmatic air or any other abnormality. He subsequently had a Computer tomography (CT) of abdomen/pelvis with contrast which showed gastrointestinal perforation with intra-abdominal free fluid.
Patient had emergency laparotomy; Perforation was noted at recto-sigmoid junction and underwent Hartman's procedure with end colostomy. Post-operatively, patient required high dependency unit care due to difficult airway secondary to epiglottis cancer. Abdominal pus sample grew mixed anaerobe spp, Clostridium sp and Coliform sp. Patient was treated with intravenous antibiotics (Co-amoxiclav, Metronidazole and Gentamicin). Histology of sigmoid colon confirmed a stercoral perforation without any evidence of dysplasia or malignancy. Histology of appendix showed serosal acute inflammation which was likely secondary to the colonic perforation. There was no evidence of dysplasia or malignancy in the histology of appendix either. Patient was stepped down to the ward for recovery after being on HDU for 2 days. Patient stoma was slow to work initially but worked eventually. After being inpatient for 5 days, 4 days post-operatively, patient recovered well enough to go home with oral antibiotics and anti-coagulation injections to complete. Patient was followed up in the outpatient clinic with the consultant. Patient then had a flexible sigmoidoscopy to assess the rectal stump prior to reversal of Hartman's 3 months later and had an elective reversal of colostomy from Hartmann's 8 months later (Figures 1,2).

\section{Discussion}

Stercoral perforation is a rare case of bowel perforation first described in 1894 by Berry J, [1], to pathological society in London. The word Stercoral means faeces and perforation 


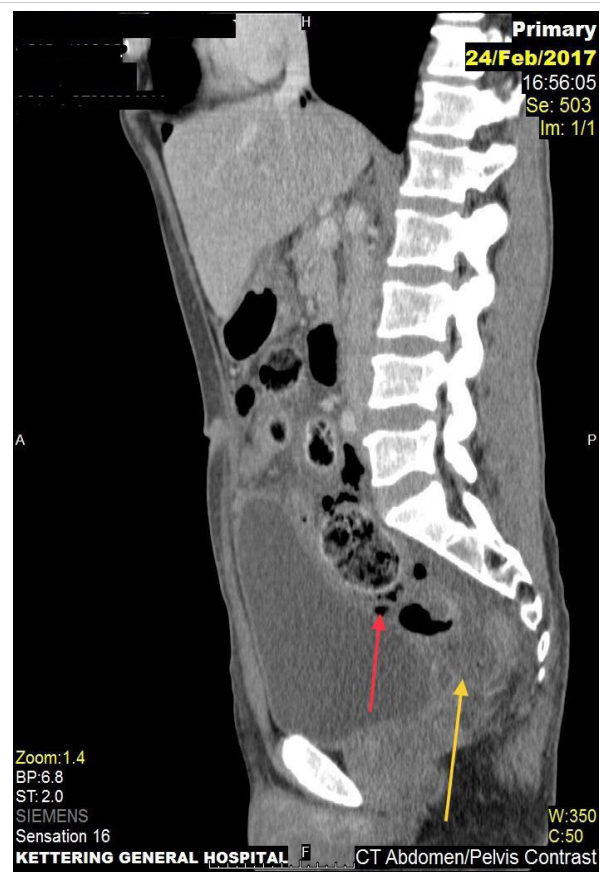

Figure 1: Axial CT (Computer Tomography) images showing red arrow indicating extra luminal air close to sigmoid colon. Yellow arrow shows free fluid collection in keeping with the sigmoid perforation.

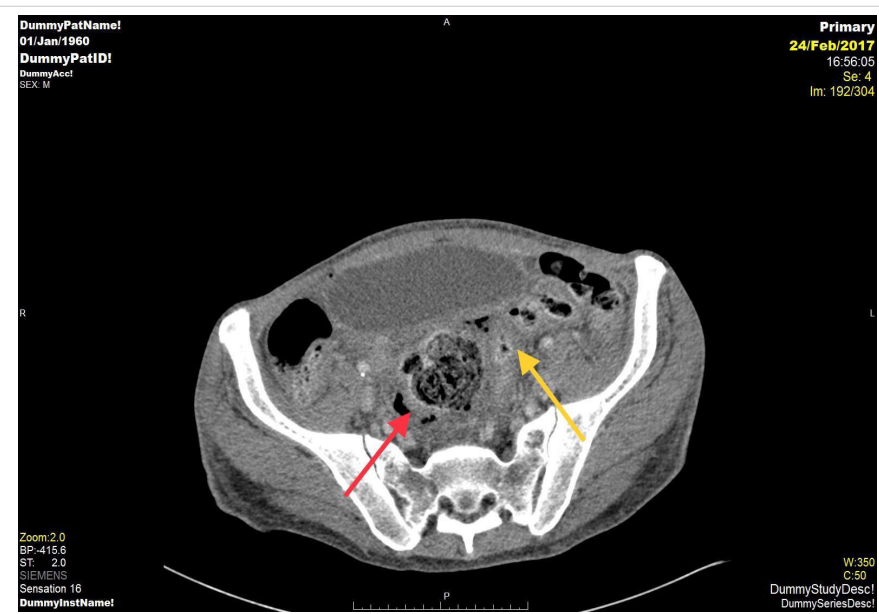

Figure 2: Sagittal section of the CT image showing red arrow indicating extra luminal air close to sigmoid colon and Yellow arrow shows free fluid collection in keeping with the sigmoid perforation.

happens due to faecal impaction causing pressure necrosis of the bowel wall secondary to ischaemia $[2,3]$. The incidence and mortality has been reported as 3.2 and $35 \%$ respectively $[3,4]$.

Maurer, et al. [4], study on 1295 patients undergoing colorectal interventions with laparotomy highlighted about the possible diagnostic criteria for Stercoral perforation based on their findings as 1) the colonic perforation was round or ovoid, exceeded $1 \mathrm{~cm}$ in diameter, and lay antimesenteric 2) Faecalomas were present within the colon, protruding through the perforation site or lying within the abdominal cavity and 3) Pressure necrosis or ulcer and chronic inflammatory reaction around the perforation site were present microscopically. The study also noted mean age as 59 years and most common site of perforation in left hemi colon and rectum [4]. Tokunaga, et al. [5], on the other hand listed the three commonest site of perforations are anterior rectum just proximal to reflection of peritoneum, Antimesenteric margin of recto-sigmoid junction and apex of sigmoid colon $[2,5]$.

Most of study in literature report antimesenteric border as the site of perforation and this could be due to the blood supply being farthest from the mesentery. Moon, et al. [6], study reported that, Faecaloma in the proximal colon with wall thickening next to perforated site are most likely due to Stercoral perforation. Whereas, Eccentric bowel wall thickening distal to the perforated site with enlarged pericolonic lymph nodes is most likely due to colorectal cancer perforation [6].

The clinical presentations of faecal impaction can range from constipation, rectal discomfort, Anorexia, Nausea, Vomiting, Pain abdomen, Faecal incontinence, Urinary frequency and urinary flow incontinence [7].

Even though, Most of the literature points to chronic constipation as the primary cause for Stercoral perforation, they have reported high risk in patients with Parkinson's suffering from constipation [8]. Use of narcotics [9], anticholinergic drugs [10], Nonsteroidal anti-inflammatory drugs (NSAID'S) [11], Patients undergoing chemotherapy [12], Ehlers-Danlos syndrome [13], Cushing's disease [14] and Psychiatric patients on Anti-Psychotic medications [15].

The most important is to have a high index of suspicion about possibility of Stercoral perforation as diagnosis in patients with chronic constipation who fail conservative measures. Early diagnosis is the key in preventing mortality reported at $34 \%$ as per a recent review [16]. Diagnostic investigations include X-ray, Ultrasound and CT of abdomen and pelvis. Taking into account the mode of presentation being acute, CT is the gold standard investigation of choice [7]. While interpreting CT, A faecaloma is considered, when the diameter of the faecal material is equal or greater than the colon and would appear as a filling defect with no stricture distally and findings of mural enhancement, thickening and sometimes lamellar calcification of the faecaloma [7]. A Gastrografin enema or manual evacuation of faeces could be attempted, if there is no CT evidence of perforation or obstructing colonic lesion. The standard treatment of choice has been resection of the diseased bowel and exteriorisation of the bowel in the form of a stoma. Huang, et al. [14], Study based on their case series reported a favourable outcome while treating Stercoral perforation depends on 1) Treatment of sepsis immediately, 2) Removal of all diseased colon, 3) Extensive peritoneal lavage, 4) Aggressive treatment of peritonitis resulting from perforation and lastly appropriate treatment of Co-morbid conditions [14]. Serpel, et al. [17], reported two cases of Stercoral perforation in the proximal part of the colon post end colostomy for Stercoral perforation within twenty four hours of operation and attributed this recurrent perforation due to loaded proximal colon and emphasised the need for intra 
operative orthograde lavage of the proximal colon to prevent recurrence of perforation [17]. Recurrence is common in the future, so prevention can be initiated by taking high fibre diet and increasing the intake of water, Avoidance of medications which affect colonic motility and usage of prokinetic agents [18].

\section{Conclusion}

Stercoral perforation should be strongly suspected in the patient population suffering with chronic constipation with risk factors including older age, use of medications (opiates, Anticholinergics, NSAID's, Anti-Psychotic medications) and presenting with faecal impaction. Prompt recognition of these patients is essential and through clinical evaluation time to time is essential in preventing a perforation, while treating them with laxatives or manual evacuation of faeces. About $10 \%$ of the diagnosis have been reported to have been missed pre-operatively [14]. Patients often being old age and on medications makes diagnosis difficult when they deteriorate, as they tend to often present with altered sensorium and chronic abdominal pain [19]. We should emphasise minimising the use of narcotics in patients suffering from chronic constipation. Early recognition of clinical deterioration and prompt use of CT would reduce mortality.

\section{References}

1. Berry J. Dilatation and rupture of sigmoid flexure short report. BR Med J. 1894; 1: 301.

2. Heffernan C, Pachter HL, Megibow AJ, Macari M. Stercoral colitis leading to fatal peritonitis: CT findings. AJR Am J Roentgenol. 2005; 184: 1189-1193.

PubMed: https://www.ncbi.nlm.nih.gov/pubmed/15788592

3. Serpell JW, Nicholls RJ. Stercoral perforation of the colon. Br J Surg. 1990; 77: 1325-1329.

4. Maurer CA, Renzulli $P$, Mazzucchelli L, Egger B, Seiler CA, et al. Use of accurate diagnostic criteria may increase incidence of stercoral perforation of the colon. Dis Colon Rectum. 2000; 43: 991-998. PubMed: https://www.ncbi.nlm.nih.gov/pubmed/10910249

5. Tokunaga Y, Hata K, Nishitai R, Kaganoi J, Nanbu H, et al. Spontaneous perforation of the rectum with possible stercoral etiology: Report of a case and review of the literature. Surg Today. 1998; 28: 937-939. PubMed: https://www.ncbi.nlm.nih.gov/pubmed/9744404

6. Moon JY, Hong SS, Hwang J, et al. Differentiation between stercoral perforation and colorectal cancer perforation. Rev Assoc Med Bras (1992). 2019; 65: 191-197.

7. Kumar $P$, Pearce $O$, Higginson A. Imaging manifestations of faecal impaction and stercoral perforation. Clin Radiol. 2011; 66: 83-88. PubMed: https://www.ncbi.nlm.nih.gov/pubmed/21147303

8. Tateno F, Sakakibara R, Aiba Y, Tsuyusaki Y, Kishi M, et al. Stercoral ulcer and colonic perforation in an individual with parkinson's disease with constipation. J Am Geriatr Soc. 2016; 64: e118-e120. PubMed: https://www.ncbi.nlm.nih.gov/pubmed/27701730

9. Tessier DJ, Harris E, Collins J, Johnson DJ. Stercoral perforation of the colon in a heroin addict. Int J Colorectal Dis. 2002; 17: 435-437.

10. Linder F, Graf W, Edholm D. Letter to the editor: Stercoral perforation of the sigmoid colon possibly associated with anticholinergic drugs or opiates: A case series. Int J Colorectal Dis. 2016; 31: 1383-1384.

11. Patel VG, Kalakuntla V, Fortson JK, Weaver WL, Joel MD, et al. Stercoral perforation of the sigmoid colon: Report of a rare case and its possible association with nonsteroidal anti-inflammatory drugs. Am Surg. 2002; 68: 62-64.

PubMed: https://www.ncbi.nlm.nih.gov/pubmed/12467320

12. Kang J, Chung M. A stercoral perforation of the descending colon. J Korean Surg Soc. 2012; 82: 125-127.

PubMed: https://www.ncbi.nlm.nih.gov/pubmed/22347716

13. Sykes EM Jr. Colon perforation in ehlers-danlos syndrome. Report of two cases and review of the literature. Am J Surg. 1984; 147: 410-413. PubMed: https://www.ncbi.nlm.nih.gov/pubmed/6367507

14. Huang WS, Wang CS, Hsieh CC, Lin PY, Chin CC, et al. Management of patients with stercoral perforation of the sigmoid colon: Report of five cases. World J Gastroenterol. 2006; 12: 500-503.

PubMed: https://www.ncbi.nlm.nih.gov/pubmed/16489660

15. Bunkar SK, Singh A, Singh RP. Stercoral perforation of the sigmoid colon in a schizophrenic patient. J Clin Diagn Res. 2015; 9: PD07-PD08. PubMed: https://www.ncbi.nlm.nih.gov/pubmed/25738027

16. Chakravartty S, Chang A, Nunoo-Mensah J. A systematic review of stercoral perforation. Colorectal Dis. 2013; 15: 930-935. PubMed: https://www.ncbi.nlm.nih.gov/pubmed/23331762

17. Serpell JW, Sen M, Giddins G, Nicholls RJ, Bradfield WJ. Stercoral perforation of the colon proximal to an end colostomy. Postgrad Med J. 1991; 67: 299-300.

PubMed: https://www.ncbi.nlm.nih.gov/pubmed/2062783

18. Hussain ZH, Whitehead DA, Lacy BE. Fecal impaction. Curr Gastroenterol Rep. 2014; 16: 404-414.

19. Gough AE, Donovan MN, Grotts J, Greaney GC. Perforated stercoral ulcer: A 10-year experience. J Am Geriatr Soc. 2016; 64: 912-914. PubMed: https://www.ncbi.nlm.nih.gov/pubmed/27100603 